Recepción: 01 / 05/ 2018

Aceptación: 15 / 08 / 2018

\title{
Estrategia de los servicios de epidemiología y la auditoría en salud
}

\author{
Strategy of epidemiology services and health audit \\ Estratégia de serviços de epidemiologia e auditoria em saúde
}

Javier I. Haro-Alvarado ${ }^{\text {I }}$

javierhaaro@gmail.com

Hortencia Manuelita-Solorzano II

hm.solorzano@gmail.com

Alba A. Sornoza-Pin III

albaapin@gmail.com

\author{
José M. Piguave-Reyes IV \\ josempr@gmail.com \\ María de los Ángeles Moreno-Cobos V \\ mdlangelesm@gmail.com \\ Petita E. Pacheco-Castro VI \\ ppcastro@gamil.com
}

Correspondencia: javierhaaro@gmail.com

I. Doctor en Medicina y Cirugía, Especialista en Auditoria Médica, Diplomado en Ciencias de la Educación para la Salud, Magister en Medicina Tropical, Docente de la Facultad de Ciencias de la Salud - Universidad Técnica de Manabí, Portoviejo, Ecuador.

II. Licenciada en Enfermería; Magister en Gerencia en Salud para el Desarrollo Local; Docente Universidad Técnica de Manabí - Escuela de Enfermería, Responsable del Departamento de Evaluación, Portoviejo, Ecuador.

III. Licenciada en Enfermería; Magister en Gerencia en Salud Sexual y Reproductiva; Docente en la Universidad Laica Eloy Alfaro de Manabí, Manta, Ecuador.

IV. Licenciado en Laboratorio Clínico; Magister en Epidemiología: Centro Especializado en Diagnóstico y Tratamiento Muñoz, Universidad de Guayaquil, Guayaquil, Ecuador.

v. Magister en Gerencia y Administración en Salud; Licenciada en Enfermería; Docente en la Universidad Laica Eloy Alfaro de Manabí, Manta, Ecuador.

VI. Licenciada en Enfermería graduada de la Universidad Estatal del Sur de Manabí, Manta, Ecuador. 


\section{Resumen}

En la presente experiencia de la estrategia de los servicios de epidemiología y la auditoría en salud, como estrategia la salud es un derecho imprescindible de las personas, puesto que para su consecución es de primordial importancia la colaboración y coordinación de los distintos sectores técnicos y sociales. La epidemiología provee muchos de los elementos para la planificación y organización de la institución de salud a fin de desarrollar una visión en conjunto del sistema optimizando el grado de satisfacción de los usuarios con la intención de la mejora continúa en el desempeño de la institución de salud hacia un crecimiento de calidad eficaz y eficiente de un sistema de salud dirigido al diagnostico y tratamiento de la enfermedad son los que permiten una mejora en la calidad de vida para todos. La auditoría médica es considerada como una herramienta de gestión clínica, no solo como medida de eficacia y eficiencia, sino también como sistema de información del comportamiento del usuario necesaria para la planeación. La responsabilidad médica consiste en la obligación del profesional de responder por eventuales daños producidos en su actuación. La responsabilidad puede ser de orden ético, moral, disciplinario, administrativo, civil y penal.

Palabras claves: Colaboración y coordinación, satisfacción de los usuarios, mejora continúa, calidad de vida, responsabilidad médica. 


\begin{abstract}
In the present experience of the epidemiology services strategy and the health audit, as a strategy, health is an essential right of the people, since the collaboration and coordination of the different technical and social sectors is of paramount importance. . Epidemiology provides many of the elements for the planning and organization of the health institution in order to develop a joint vision of the system optimizing the degree of satisfaction of users with the intention of continuous improvement in the performance of the health institution towards an effective and efficient quality growth of a health system aimed at the diagnosis and treatment of the disease are those that allow an improvement in the quality of life for all. The medical audit is considered as a clinical management tool, not only as a measure of effectiveness and efficiency, but also as an information system of the user behavior necessary for planning. The medical responsibility consists of the obligation of the professional to answer for possible damages produced in his performance. The responsibility may be ethical, moral, disciplinary, administrative, civil and criminal.
\end{abstract}

Keys words: Collaboration and coordination, user satisfaction, continuous improvement, quality of life, medical responsibility. 


\section{Introducción.}

La estrategia como instrumento y propuesta para el perfeccionamiento de las competencias profesionales de la medicina considera acciones relacionadas con el diagnóstico basado en evidencias para revisar y difundir los ensayos clínicos, evaluar las nuevas tecnologías, analizar la toma de decisiones y revisar las políticas institucionales para una mejora continua asistencial, forman parte de los objetivos, en la búsqueda del método de los mejores servicios de epidemiología, así como la auditoria de calidad en salud.

El punto de partida para este proceso lo fue en primer lugar la Epidemiología, la cual marca esencialmente cómo debieran ser conducidos los sistemas y servicios de salud. Dussault, (1995).

Con respecto a la prestación de servicio en salud ayuda a establecer las necesidades de los usuarios con eficiencia para que logren ser recompensadas cuando lo solicitan, es la forma de hacer las cosas que corresponde a toda la institución. La introducción de políticas de calidad en los servicios sanitarios es una herramienta de mejora utilizada ampliamente, probada también en otros servicios públicos. Los servicios de salud pública se encuentran en una posición peculiar dentro del sistema de salud, y algunas de las innovaciones de gestión introducidas en los servicios asistenciales han tardado más en aplicarse en los servicios de salud pública, Rivadeneyra (2008).

El estudio de las enfermedades como fenómenos poblacionales es casi tan antiguo como la escritura, y las primeras descripciones de padecimientos que afectan a poblaciones enteras se refieren a enfermedades de naturaleza infecciosa. El papiro de Ebers, que menciona unas fiebres pestilentes - probablemente malaria- que asolaron a la población de las márgenes del Nilo alrededor 
del año 2000 a.C., es probablemente el texto en el que se hace la más antigua referencia a un padecimiento colectivo. Cartwright y Biddiss, (1972).

La aparición de plagas a lo largo de la historia también fue registrada en la mayor parte de los libros sagrados, en especial en la Biblia, el Talmud y el Corán, que adicionalmente contienen las primeras normas para prevenir las enfermedades contagiosas. De estas descripciones, destaca la de la plaga que obligó a Mineptah, el faraón egipcio que sucedió a Ramsés II, a permitir la salida de los judíos de Egipto, alrededor del año 1224 a.C., La Santa Biblia, (1960).

Según Winslow, (1943), la aparición de la pandemia de peste bubónica o peste negra que azotó a Europa durante el siglo XIV (de la cual se dice que diariamente morían 10 mil personas), finalmente condujo a la aceptación universal -aunque todavía en el ámbito popular- de la doctrina del contagio.

Georgius Agrícola: Maestro y científico alemán, considerado el padre de la mineralogía, escribió el libro “De Re Metallica” (1556), donde hace un análisis sistemático de la minería y la metalurgia en el Siglo XVI en Checoslovaquia, describiendo con gran detalle las prácticas mineras usadas y los efectos de los metales en los mineros. Bernardino Ramazzini: Médico Italiano (Siglo XVII - XVIII), considerado Padre de la Medicina Ocupacional, describió las enfermedades relacionadas con el trabajo y sus causas en el libro "De Morbis Artificum" (1790). Pulido, (2010).

La investigación realizada en el campo de la epidemiología experimentó durante el siglo XIX un extraordinario avance, especialmente con los trabajos de Robert Storrs (1840), Oliver Wendell Holmes (1842) e Ignaz Semmelweis (1848) sobre la transmisión de la fiebre puerperal; los de P.L. Panum (1846) sobre la contagiosidad del sarampión; los de Snow (1854) sobre el modo de 
Javier I. Haro-Alvarado; Hortencia Manuelita-Solorzano; Alba A. Sornoza-Pin; José M. Piguave-Reyes; María de los Ángeles Moreno-Cobos; Petita E. Pacheco-Castro

transmisión del cólera, y los de William Budd (1857) sobre la transmisión de la fiebre tifoidea. La importancia de estos trabajos radica en el enorme esfuerzo intelectual que estos investigadores debieron hacer para documentar -mediante la pura observación- propuestas sobre la capacidad transmisora, los mecanismos de contagio y la infectividad de agentes patógenos sobre los que aún no podía demostrarse una existencia real. López, et al., (2000).

En los años 1830 a 1860 aparece el Movimiento de Salud Pública, el que logra generar sistemas de estadísticas poblacionales y registros de defunciones en Europa y los primeros estudios estadísticos de salud de trabajadores. Entre estos estudios tenemos los estudios de William Farr (Inglaterra), reconocido fundador del concepto moderno de vigilancia y, los de Villermé (Francia) quien realizó estudios epidemiológicos acerca de la industria francesa en la vida de los obreros. En los años 1900 a 1940 se hace evidente la profesionalización de las actividades productivas, el sindicalismo y la intervención gubernamental. Ello logra, entre otros, capacitación formal en medicina e higiene industrial, regulación del trabajo entre niños y mujeres, y normas para industrias peligrosas (minería). Pulido, (2010).

La epidemiología ocupacional cuantitativa moderna se remonta a la segunda mitad del siglo pasado. En ella los ritos más importantes son: 1950, Gran Bretaña: Método de cohortes históricas. Richard Doll, publicó el primer estudio epidemiológico longitudinal detallado sobre 113 trabajadores del sector textil-asbesto, seguidos durante $20 \quad$ años. 1960, Estados Unidos: Estudios de mortalidad en industrias de acero, asbestos, goma y minería. 1970-1980: Evaluación cuantitativa de exposición; avances en métodos estadísticos. Pulido, (2010).

La historia de la auditoría médica se remonta a 1910, cuando aparece el informe Flexner respecto a la enseñanza de la Medicina en las diferentes universidades en USA, criticando 
severamente el sistema existente, y que concluyó en la no-acreditación de 47 facultades de Medicina. En 1917 el Colegio Americano de Cirujanos dicta las Normas para la acreditación hospitalaria. En 1950, se realiza un estudio piloto en 15 hospitales, institucionalizándose el procedimiento, venciendo el temor y la suspicacia de considerar a la auditoría como un instrumento de control y punición. Garaycochea (2000).

La auditoría médica nació en los hospitales norteamericanos en 1918, iniciándose con un sistema de acreditación de hospitales por el Colegio de Cirujanos, colocando especial énfasis, en aquella época, en el perfeccionamiento de las historias clínicas, Solar, (1960).

Entender a la auditoría como una herramienta de gestión de la calidad y, a su vez, como una herramienta de la gestión gerencial, implica que la auditoría y la organización, así como los auditados deben conocer los elementos básicos que determinan las etapas crecientemente evolutivas que han caracterizado el desarrollo del arte, así como saber utilizar las metodologías e instrumentos propuestos en las experiencias exitosas, armonizándolas con sus condiciones específicas y adaptándolas de manera responsable y creativa. Ministerio de protección social, (2007).

La auditoría del servicio nace de la convicción de que el cliente es lo más importante y es un elemento insustituible para el éxito de cualquier empresa productiva o de cualquier otra entidad que, aún sin fines de lucro quiere impresionar favorablemente a sus usuarios, con el propósito de que cada intervención sea una experiencia memorable; La auditoría del servicio, es un conjunto de herramientas innovadoras y técnicas que permiten conocer, cosas como: ¿Cuál es la percepción del producto o servicio que ofrecemos?, ¿Cómo es nuestro posicionamiento, respecto a nuestra competencia?, ¿Qué es lo que el cliente espera de nosotros?, ¿Por qué perdemos clientes? y ¿Por qué nuestra competencia crece más que nosotros?. Cifuentes, (2015). 
Javier I. Haro-Alvarado; Hortencia Manuelita-Solorzano; Alba A. Sornoza-Pin; José M. Piguave-Reyes; María de los Ángeles Moreno-Cobos; Petita E. Pacheco-Castro

El concepto de auditoría médica y el de epidemiología son distintos: El primero hace referencia a la búsqueda de las cualidades del servicio o de un producto, que hace el hombre para satisfacer sus necesidades de bienestar, el segundo es un método que permite explorar la frecuencia y distribución de las enfermedades, sus causas y los aspectos para prevenirlas. Las herramientas del método epidemiológico permiten obtener estrategias confiables para medir la calidad con un nivel científico adecuado. Álvarez y Álvarez, (2009).

La epidemiología se destaca en la actualidad como una metodología de apoyo al trabajo de los auditores en salud. El conocimiento de los elementos epidemiológicos, de los elementos de calidad y auditoría, y de sus interrelaciones, se torna indispensable para la mayoría de los auditores que poseen una gran valencia en estos temas. Álvarez y Álvarez, (2009).

\section{Metodología.}

En la presente exploración científica se realizo mediante un análisis de la bibliografía de la literatura más cercana sobre la estrategia de los servicios de epidemiología y la auditoría en salud. Para la realización de la actual investigación, se considero, información confiable sobre trabajos plasmados, tratados en este tema, se utilizo información bibliográfica, consultas electrónicas, misma que se la obtuvo de libros electrónicos, artículos científicos y revistas.

\section{Desarrollo.}

La prestación de servicio en atención al usuario o cliente

El servicio se define como "un producto primordial" de la calidad y confiabilidad en la atención, el servicio no es una cosa "unidimensional" lo que está en el meollo del argumento, respecto a que el servicio es tanto una mercancía como lo es un automóvil y necesita tanto de la 
administración como de un estudio sistemático. Se puede dividir esta definición del servicio en dos, el servicio paralelo, lo que se define como garantía y se maneja como la asistencia técnica y, el servicio como valor agregado, lo que se especifica como dar más de lo que espera el cliente, en definitiva buscar la satisfacción total, Albrecht, \& Zemke, (1988) y Berry, (2002).

El servicio nos da unas pautas para mirar al personal como uno de los elementos en consecuencia de la calidad del servicio. El enfoque norteamericano incluía dos lineamientos de acción: el primero es el departamento atención al cliente y el de quejas y reclamos; al contrario de la filosofía de la gerencia de servicio donde todo el mundo tiene que desempeñar un papel y se asegura de que todo salga bien para el cliente, este enfoque busca crear una cultura de servicio que haga que la atención al cliente sea excelente y tiene una misión reconocida para todos incluso el gerente, Alberto, et al., (1988) y Guerrero, et al., (2007).

Atención de salud se define como el conjunto de servicios que se prestan al usuario en el marco de los procesos propios del aseguramiento, así como de las actividades, procedimientos e intervenciones asistenciales en las fases de promoción y prevención, diagnóstico, tratamiento y rehabilitación que se prestan a toda la población. La calidad de la atención de salud es la provisión de servicios de salud a los usuarios individuales y colectivos de manera accesible y equitativa, a través de un nivel profesional óptimo, teniendo en cuenta el balance entre beneficios, riesgos y costos, con el propósito de lograr la adhesión y satisfacción de dichos usuarios, Minsalud, (2013).

Con respecto al usuario el servicio para él es la gestión que realiza cada persona que trabaja en una institución y que tiene la oportunidad de estar en contacto con los usuarios generar en ellos algún nivel de satisfacción, agilidad, claridad y pertinencia, enfocar los servicios ofrecidos y las comunicaciones hacía aquellos usuarios que más lo requieren, ayuda a determinar las necesidades de 
Javier I. Haro-Alvarado; Hortencia Manuelita-Solorzano; Alba A. Sornoza-Pin; José M. Piguave-Reyes; María de los Ángeles Moreno-Cobos; Petita E. Pacheco-Castro

los usuarios con eficiencia para que puedan ser satisfechas cuando lo solicitan, es la forma de hacer las cosas que compete a toda la institución. Cifuentes, B., (2015)

Factores claves del servicio al cliente o usuario

Así mismo, los servicios centrados en el usuario continúan valorando la pericia de los profesionales de salud, los prestadores de servicios poseen las habilidades y los conocimientos especializados para ayudar al usuario a tomar decisiones informadas, para garantizar la seguridad del usuario y para mantener la calidad técnica de atención. Atención centrada en el usuario, (s/f).

En todo lugar, las personas continuamente evalúan la calidad de los servicios que reciben, dada la opción, ellas escogen prestadores de servicios e instalaciones que ofrecen la mejor atención disponible, según la percibe ¿Cómo juzgan los clientes la calidad de los servicios de salud? Cada vez más investigaciones están descubriendo qué desean los clientes y cómo medir la satisfacción del cliente. Tanto en los países desarrollados como en los países en desarrollo, los clientes buscan la satisfacción total de los servicios, Atención centrada en el usuario, (s/f) y Ginocchio, (2011).

Conocer el grado de satisfacción de los usuarios en relación con los servicios de salud resulta importante, especialmente para aquellas instituciones de salud que decidieron implementar el Sistema Único de Acréditación, que las destaca como las de mejor calidad en la prestación de servicios y que deben, por tanto, verificar el impacto que ejercen sobre la satisfacción del cliente. Evaluar la satisfacción de los usuarios es un proceso complejo, entre otras razones por la alta subjetividad que presenta. Rodríguez y Lozano, (2012).

\section{Epidemiología}


La epidemiología es la ciencia que trata del estudio de la distribución de las enfermedades, de sus causas y de los determinantes de su frecuencia en el hombre, así como del conocimiento de la historia natural en las enfermedades y del conocimiento de datos para una intervención orientada al control o erradicación de ellas. Su práctica se realiza a través del "método epidemiológico", basado en la observación de los fenómenos, la elaboración de hipótesis, el estudio o experimentación de éstas y la verificación de los resultados. Se ha dicho que es "una ciencia de lo poblacional o colectivo (como la sociología) pero construida del modo de las ciencias biológicas o naturales". En éste sentido, se describen tres etapas o fases del método epidemiológico: descriptivo, analítico y experimental, Orlando, (2005).

Epidemiología Es la disciplina científica que estudia los factores que tienen efecto sobre la salud y la enfermedad de una población, se utiliza para diseñar, monitorear y evaluar intervenciones de salud pública y tomar decisiones de administración sanitaria. Persona Lugar Tiempo. Estudia la ocurrencia, distribución, factores causales y de riesgo, que afectan los problemas de saludenfermedad de la comunidad, bien sea esta de origen genético, infeccioso, degenerativo o cualquier otro. Manrique, (2015).

La Epidemiología es considerada la ciencia básica de la salud pública. A su vez, la Salud Pública es parte de las políticas sociales que un Estado debe desarrollar a favor de la población, por tanto, es deseable que las decisiones se sustenten en la compresión científica del estado de salud de dicha población para respaldar la implementación de modelos, planes, proyectos y/o programas de gran impacto social. Epidemiología \& salud pública. Manrique, (2015).

Epidemiología, Es la disciplina científica que estudia los factores que tienen efecto sobre la salud y la enfermedad de una población, se utiliza para diseñar, monitorear y evaluar intervenciones 
Javier I. Haro-Alvarado; Hortencia Manuelita-Solorzano; Alba A. Sornoza-Pin; José M. Piguave-Reyes; María de los Ángeles Moreno-Cobos; Petita E. Pacheco-Castro

de salud pública y tomar decisiones de administración sanitaria. Estudia la ocurrencia, distribución, factores causales y de riesgo, que afectan los problemas de salud-enfermedad de la comunidad, bien sea esta de origen genético, infeccioso, degenerativo o cualquier otro. Aplica permanentemente el estudio del método científico a fin de establecer y desarrollar los procedimientos, mecanismos, acciones e intervenciones pendientes a promover y mejorar la salud. Manrique, (2015).

\section{Tipos de Epidemiología}

Epidemiología descriptiva, Describe el fenómeno epidemiológico en tiempo, lugar y persona, cuantificando la frecuencia y distribución del fenómeno mediante medidas de incidencia, prevalencia y mortalidad, con la posterior formulación de hipótesis.

Epidemiología analítica Es la disciplina científica que estudia la distribución y determinantes de las enfermedades y daños a la salud de una población durante un periodo de tiempo para aplicar medidas de control. Busca establecer posibles Relaciones causales entre factores a los que se exponen personas y poblaciones y las enfermedades que presentan. Las medidas empleadas son los factores de riesgo, cuyo resultado es una probabilidad: Riesgo Absoluto (probabilidad de una enfermedad) y Riesgo Relativo (comparación de dos riesgos absolutos entre sí). Manrique, (2015).

\section{Aplicación de la Epidemiología en la Planificación de Servicios}

Según Orlando, (2005). Se basa en la medición de las necesidades de la población a través del conocimiento de la demanda. De esta manera se utilizan los indicadores de demanda como expresión de las personas que reciben atención o que desean recibirla. Este indicador expresa la necesidad cuando los servicios se distribuyen con equidad, en forma homogénea, para toda la población, y ésta posee una semejante accesibilidad a dichos servicios siendo, por lo demás 
aceptablemente satisfactorios los registros de las prestaciones médicas. Cuando ello no es así, el perfil de la salud puede ser distorsionado; tal es el caso cuando la demanda resulta un producto de la oferta o cuando se produce un sesgo contra los niveles socioeconómicos más descendidos. Por ello no se puede tomar la demanda de la atención como indicador de morbilidad y, en consecuencia, de necesidad: puede ser engañosa.

En cada etapa de la Planificación, los hechos deberían ser investigados e interpretados con metodología y criterio epidemiológico para la toma de decisiones. De esta manera, durante las etapas de diagnóstico, decisión y evaluación, la epidemiología será responsable de planear las necesidades, estimar los daños, valorar la importancia relativa de los fenómenos y determinar las opciones que existan para el curso de las acciones posibles. Valorando por último, el resultado de las mismas. Así se llegará a la toma de decisiones con la presentación del problema racionalmente estudiado y priorizado de acuerdo con las posibles opciones en términos de costo-beneficio, Orlando, (2005).

Usos de la Epidemiología. 1. Medición y análisis de la salud de poblaciones. 2. Descripción de la historia natural de la enfermedad 3. Identificación de los determinantes de las enfermedades 4. Control y prevención de la enfermedad 5. En la selección de métodos de control y prevención: 6 . Planificación y evaluación de servicios de salud 7. Misceláneos. 8. Lectura crítica de información científica. 9. Predicción de escenarios sanitarios. 10. Estudio de la forma en que se distribuyen recursos de acuerdo a las necesidades de la población. 11. Aplicación de sus métodos al escenario clínico. Manrique, (2015).

La epidemiología puede y debe ser utilizada a los efectos de la administración de los servicios de salud. Contribuye a la elaboración del diagnóstico de una comunidad respecto de la 
Javier I. Haro-Alvarado; Hortencia Manuelita-Solorzano; Alba A. Sornoza-Pin; José M. Piguave-Reyes; María de los Ángeles Moreno-Cobos; Petita E. Pacheco-Castro

exigencia, naturaleza y distribución de la salud y la enfermedad. Constituye un medio para controlar la salud de la población. Mas aún, utilizando los datos causales obtenidos por los epidemiólogos clásicos, los administradores pueden identificar individuos en situación de riesgo, su mercado o población objetivo potencial. Orlando, (2005).

La epidemiología provee muchos de los elementos para la administración y planificación de los servicios de salud, y para su evaluación. Administración, Proceso mediante el cual se supervisa la producción de los servicios (en este caso el de salud). Funciones: Planificación, organización, dirección, coordinación y control; De la unión de estas funciones surge la toma de decisiones sobre la base de información. Orlando, (2005).

\section{Vigilancia epidemiológica e inteligencia sanitaria}

Vigilancia Epidemiológica, Proceso continuo y sistemático de colección, análisis e interpretación de datos de las enfermedades o daños sujetos a notificación, para conocer su tendencia, evolución, identificar las regiones geográficas y los grupos poblacionales más comprometidos, conocer el estado de salud actual de la población, identificar precozmente los brotes o epidemias para su oportuna intervención y control. Es información analizada para la acción y su objetivo es obtener información actualizada y oportuna para ofrecer orientación técnica a quienes tienen responsabilidad de decidir acciones de prevención y control. Manrique, (2015).

En salud laboral, el concepto de vigilancia epidemiológica se refiere a $<<$ la recogida sistemática y análisis de información sobre los factores de riesgo laborales (exposición) o sobre los efectos en la salud (enfermedades, accidentes) para la observación de la distribución y tendencia de los fenómenos de interés, con el fin de identificar los problemas y de dirigir y evaluar las intervenciones preventivas $>>$. Rantanen, et al. (2001). 
Sistema de vigilancia epidemiológica. Programa de acción conformado por un conjunto de estrategias y acciones que permiten identificar y detectar los daños y riesgos para la salud. Su importancia radica en la capacidad de generar información útil para la orientación de los programas, las intervenciones que se requieren en la atención de los padecimientos, situaciones de riesgo que afectan a la comunidad en forma grave y frecuente. Manrique, (2015).

La vigilancia epidemiológica es una herramienta que convierte a la prevención de los riesgos laborales y la salud en el trabajo en un proceso dinámico y de mejora continua. Dicha vigilancia se constituye como un instrumento de alerta y de información para la acción y utiliza los mismos ingredientes de conocer, actuar, evaluar y reajustar que utilizan los procesos de mejora continua desarrollados en el campo de los sistemas de gestión de calidad. Maizlish, (2000).

La planificación y la evaluación de las acciones son actividades que forman parte de la vigilancia colectiva que sirve al proceso de mejora continua proporcionando información necesaria para la planificación y la evaluación de las intervenciones. Es, por lo tanto, una herramienta básica para la gestión de la prevención de los riesgos y la salud en el trabajo. En todo modelo de planificación se necesita saber de dónde se parte, a dónde se quiere llegar y con qué medios. En primer lugar, la vigilancia colectiva debe aportar información válida para el diagnóstico de la situación de partida. Los métodos y técnicas que se han comentado en esta guía van a proporcionar al servicio de prevención indicadores de los problemas en un momento determinado. Urbaneja, et al. (2015).

Inteligencia Sanitaria Proceso analítico-sintético que permite caracterizar, medir y explicar el perfil de salud-enfermedad de una población, incluyendo los daños y problemas de salud, así como sus determinantes, con el fin de identificar necesidades y prioridades en salud, los cuales son 
Javier I. Haro-Alvarado; Hortencia Manuelita-Solorzano; Alba A. Sornoza-Pin; José M. Piguave-Reyes; María de los Ángeles Moreno-Cobos; Petita E. Pacheco-Castro

de utilidad para la definición de intervenciones y estrategias, conocido como el Análisis de Situación de Salud. Selección y presentación de información a los agentes-profesionales clave, en forma tal que lleve a acciones que modifiquen la salud de los pacientes y de las poblaciones. Se emplea para la búsqueda de la mejor respuesta a los problemas de salud. Se considera los 3 niveles de gestión: El macro (internacional y nacional). El meso (región y área). El micro (en la consulta del médico). Manrique, (2015).

\section{Epidemiología y auditoría}

Los conceptos de epidemiología y de auditoría médica son diferentes: El primero es un método que permite explorar la frecuencia y distribución de las enfermedades, sus causas y los aspectos para prevenirlas. Se diferencian pero se complementan. Las herramientas del método epidemiológico permiten obtener estrategias confiables para medir la calidad con un nivel científico adecuado; el segundo hace referencia a la búsqueda de los atributos o cualidades de calidad de un producto o del servicio de salud, que hace el hombre para satisfacer sus necesidades de bienestar. Álvarez y Álvarez, (2009).

Epidemiología \& Auditoría médica, La Epidemiología permite obtener estrategias confiables para medir la calidad con un nivel científico adecuado. Promover las estrategias instrumentales de la Medicina Basada en la Evidencia para revisar y difundir los ensayos clínicos, evaluar las nuevas tecnologías, analizar la toma de decisiones y revisar las políticas institucionales para la mejora asistencial, desde una perspectiva de calidad y seguridad de la atención en salud (sanitaria). Manrique, (2015).

Promueve las estrategias instrumentales de la Medicina basada en la evidencia para revisar y difundir los ensayos clínicos, evaluar las nuevas tecnologías, analizar la toma de decisiones y revisar 
las políticas institucionales para la mejora asistencial, son objetivos, tanto de la auditoría de calidad como el de la epidemiología. Álvarez y Álvarez, (2009).

La epidemiología se destaca en la actualidad como una metodología de apoyo al trabajo de los auditores en salud. El conocimiento de los elementos epidemiológicos, de los elementos de calidad y auditoría, y de sus interrelaciones, se torna indispensable para la mayoría de los auditores que poseen una gran valencia en estos temas. Álvarez y Álvarez, (2009).

Los conceptos de epidemiología como los de auditoría médica, abarcan un importante renglón para profesionales que se desempeñan en tareas técnico-sanitarias, como auditores de salud, planificadores o seguimiento de programación con el fin de investigar adecuadamente como auditores de calidad en salud. Los auditores médicos de calidad se les presenta con frecuencia la necesidad de emplear algunas metodologías epidemiológicas, lo hacen sin que conozcan los principios de su aplicación, porque no poseen entrenamiento básico para la evaluación de procesos, programas o de construcción de indicadores de salud, propios de disciplinas como la epidemiología. Álvarez y Álvarez, (2009).

\section{Auditoría de la atención en salud}

La auditoría de la atención de salud, al trabajar sobre la base de registros de lo efectuado, además de evaluar la calidad de estos, valora también la calidad de la atención misma, demostrando que hay una relación directa entre la calidad de los registros y la de la atención prestada. La sistematización actual de la calidad en auditoría considera el concepto de control de calidad, el cual consiste en que el producto o servicio se adecua a las especificaciones determinadas previamente. Osorio, et al. (2002). 
La auditoría del servicio se define como el conjunto de estrategias diseñadas de manera ordenada y consecuente para que el cliente evalúe la calidad y los niveles de satisfacción del servicio recibido dentro del ciclo de la prestación y de acuerdo con modelos de excelencia ya definidos. El cliente se interesa por conseguir la satisfacción de sus necesidades, su lealtad está delimitada por la calidad del servicio, en el momento en que el nivel de servicio esté por debajo de su expectativa la lealtad se desvanece y orienta buscando otra opción, por esto el nivel del servicio debe ser permanentemente satisfactorio. Cifuentes, (2015).

La auditoría médica es un proceso interdisciplinario, que permite al Cuerpo Médico realizar la evaluación del acto médico, con los objetivos de:

- Mejorar la práctica médica,

- Ser un medio de educación continua, y

- Mejorar la calidad de la atención médica. Garaycochea (2000).

¿Cuál es la interrelación entre estos temas y cuál es el apoyo que presta la epidemiología a la auditoría médica? Su respuesta es amplia: La epidemiología es una disciplina que emplea el método científico como elemento central de su trabajo, mientras la auditoría médica, busca información que permita conocer la situación de calidad de una institución o de un proceso para el establecimiento de planes de mejoramiento, de tal manera que la epidemiología es útil en la elaboración de los instrumentos para recolección de información, conocimiento de los diseños de investigación, la elaboración de indicadores, el uso de escalas. Álvarez y Álvarez, (2009).

\section{La auditoría médica: herramienta de gestión}


Según Osorio, et al. (2002). Campos de acción de la auditoría médica. La auditoría médica es considerada actualmente como una herramienta de gestión clínica, que puede abarcar diferentes aspectos del quehacer médico asistencial, administrativo y financiero-contable:

- Aspectos médico legales. En este campo se actúa con el sentido de prevenir la ineficiente práctica médica, cautelando además el cumplimiento de las normas legales y de las disposiciones sanitarias vigentes, actuando en estrecha relación y colaboración con los Departamentos de Asesoría Jurídica.

- Aspectos normativos, íntimamente relacionados con el punto anterior, la que estimula la elaboración, revisión y readecuación de normas, pautas y manuales de procedimientos que regularicen el grado de cumplimiento y sujeción.

- Aspectos técnicos evaluativos a través de la valoración de los actos y conductas; implica realizar un control de la calidad de éstos.

- Aspectos éticos, al respecto corresponde supervisar el fiel cumplimiento de las normas éticas y morales en las conductas de las personas, la denominada deontología médica, que establece el deber ser del actuar médico, complementándose en este aspecto con las funciones del Comité de Ética de cada establecimiento.

- Aspectos financieros administrativos y de mercado. También tiene incidencia en este campo, compatibilizando una adecuada relación entre eficiencia, costos, calidad y seguridad de la atención brindada.

- Aspectos docentes y de investigación que estimulan la enseñanza y perfeccionamiento continúo de post grado, teniendo efectos educativos y preventivos que permiten la obtención de aprendizaje y experiencia. 
Javier I. Haro-Alvarado; Hortencia Manuelita-Solorzano; Alba A. Sornoza-Pin; José M. Piguave-Reyes; María de los Ángeles Moreno-Cobos; Petita E. Pacheco-Castro

La auditoría médica se define como una evaluación crítica y periódica de la calidad de la atención médica que reciben los pacientes, mediante la revisión y el estudio de las historias clínicas y las estadísticas hospitalarias. Su propósito fundamental es procurar que el enfermo reciba la mejor atención médica posible y su objetivo específico es elevar su calidad. Moya, (1968).

La Auditoría Médica también conocida como Evaluación Médica es un proceso de investigación y evaluación de la calidad de atención médica, que puede ser realizada en 2 formas: lo. - Auditoría Interna, la cual es realizada por los sectores de salud tal como lo indica su nombre en forma interna o sea una autoevaluación; y 2o. - Auditoría Externa que es la realizada por comisiones o departamentos sin relación directa con el sector de salud o médico evaluado. Alvarado, (1986).

La auditoría médica es un instrumento fundamental para mejorar la calidad de la atención ofrecida a los pacientes, y que responde a las siguientes preguntas:

¿Qué atención brindamos?

¿Hicimos lo que proyectamos hacer?

¿Qué deberíamos haber hecho?

¿Hacemos lo que debemos hacer?

¿Podemos mejorar lo que hacemos?

La misión de la auditoría médica es garantizar las habilidades técnicas de los profesionales, permitir el uso eficiente de los recursos disponibles, y lograr la satisfacción del paciente en sus demandas y expectativas. La auditoría cumple un ciclo que comprende desde el establecimiento de 
criterios y estándares, la revisión de historias clínicas y colección de datos, resultados y análisis de los hallazgos, implementación de cambios, y por último monitoreo de los cambios, cerrándose y repitiéndose el ciclo nuevamente. Garaycochea (2000).

\section{El trabajo de auditoría}

El auditor interno en el desempeño de su actividad profesional debe cumplir una serie de requisitos: Los más relevantes son su independencia de las actividades que audita; el apoyo de los directivos para ejecutar su trabajo en forma libre y sin interferencias; la objetividad, vale decir, mantener una cierta actitud mental, que le permita tener la honesta convicción que en el producto de su trabajo no exista nada que comprometa la calidad de su labor; debe tener conocimientos, habilidades y destrezas en la aplicación de las normas, procedimientos y técnicas requeridas para la auditoría; ética profesional, para cumplir con normas profesionales de conducta que le imponen patrones de honestidad, objetividad, diligencia y lealtad. Contraloría General de la República de Chile, (1996).

En el proceso de auditoría se han delineado cuatro etapas básicas, que deben estar materializadas en un programa escrito. En la planificación se determinan los objetivos y el alcance de la auditoría; las actividades a analizar, los recursos necesarios para efectuar el estudio, análisis de terreno, identificación de áreas críticas, etc. El examen y evaluación de la información es la fase en la que todos los datos seleccionados se recogen, interpretan y analizan. La presentación de resultados da origen a las conclusiones y recomendaciones. Posteriormente se hace entrega de informes escritos del resultado y se divulga. En la fase de seguimiento se determina si las acciones correctoras se aplican o no y si éstas alcanzan los objetivos deseados. Osorio, et al. (2002).

\section{Historia clínica en la auditoría médica}


Son fuentes de información de la Auditoría Médica la Historia clínica, los protocolos de diagnóstico y tratamiento, y los protocolos de exámenes de ayuda diagnóstica. La revisión sistemática de la Historia Clínica, su confrontación con protocolos de diagnóstico y tratamiento, constituye la piedra angular de la auditoría y contribuye a retroalimentar el conocimiento médico, facilitando la superación de inconsistencias para lograr una buena calidad de atención médica. Garaycochea (2000).

Para la realización de una auditoría médica el documento esencial es la ficha clínica. Ésta junto a las estadísticas hospitalarias, normas, protocolos, padrones estándares y la labor del auditor, permiten un análisis acucioso del trabajo médico. La ficha clínica es el documento en el cual se registra la totalidad de las prestaciones médicas recibidas por el enfermo, los exámenes realizados, además de los solicitados y todo aquello de lo que se requiere dejar constancia, con relación a la patología del paciente y a las acciones desarrolladas para obtener su curación. Osorio, et al. (2002).

Según Garaycochea (2000). La historia clínica es uno de los elementos fundamentales para el trabajo médico, y es la pieza clave en toda auditoría médica. La historia clínica es importante por cuanto contiene datos acerca de lo que se ha encontrado en un paciente, lo que se ha pensado y lo que se ha hecho por su atención. Sin embargo los datos de la historia clínica pueden tener varios propósitos:

1. Es un instrumento para trasmitir la información acerca de un paciente, permitiendo su conocimiento por varios especialistas, convirtiéndose en relato sobre la biografía médica del paciente. 
2. Un segundo propósito de la historia clínica es la investigación científica, sirviendo como elemento base para la colección de datos con respecto a diagnóstico, tratamiento, o procedimientos realizados.

3. Se emplean además las historias clínicas en el campo administrativo, con muchos propósitos entre ellos el pago de servicios prestados, por ejemplo.

4. La historia clínica es un instrumento médico legal, especialmente en casos derivados del ejercicio inadecuado de la profesión.

La historia clínica es el instrumento más útil para valorar la calidad de atención médica brindada a los pacientes, pues es la biografía médica de ellos. Por estas razones los datos en las historias clínicas debieran estar debidamente organizados. La organización de los datos va a depender del enfoque, existiendo autores como Weed que refieren que la historia clínica debe estar orientada hacia los problemas del paciente, que facilita el trabajo médico y la comunicación entre especialistas. Garaycochea (2000).

La ficha clínica como instrumento y por constituir un medio de prueba judicial que influye en el proceso, debe reunir ciertos requisitos básicos, tales como: Uso de letra clara y legible, los registros deben ser completos, oportunos y pertinentes, la información debe ser clara, concisa y ordenada para que permita un análisis eficiente. Es importante considerar que lo que no está escrito se supone no realizado. Es un documento reservado, característica que surge de la propia esencia de ella y en lo que atañe al médico se encuentra protegida por el secreto profesional. Osorio, et al. (2002). 
Auditoría médica y responsabilidad médica. La responsabilidad médica consiste en la obligación del profesional de responder por eventuales daños producidos en su actuación. La responsabilidad puede ser de orden ético, moral, disciplinario, administrativo, civil y penal. Esta última es la que proviene de una acción u omisión tipificada como infracción del código penal. En relación con el protocolo de derechos del paciente en el que se entrega una sistematización de preceptos legales que explicitan los derechos de los pacientes en materia de atención de salud, es muy importante, abordar la responsabilidad médica, desde el punto de vista del derecho en diversos aspectos. Osorio, et al. (2002).

\section{Conclusiones.}

Como estrategia la salud es un derecho imprescindible de las personas, puesto que para su consecución es de primordial importancia la colaboración y coordinación de los distintos sectores técnicos y sociales. No existe un cúmulo de datos que pueda responder a todas las necesidades de la epidemiología, pero si es posible puntualizar la información del diagnostico, lo que permite orientar la acción y el hecho que sea necesario para planificar la gestión de calidad. El encuentro de los problemas es la pauta para el establecimiento de un cuadro de acción a fin de proponer soluciones de salud para todos.

Las medidas de promoción y prevención en la salud son la que van a permitir un mayor grado de efectividad frente a los hábitos de vida a fin de lograr modificarlos ya que son la base causal de la enfermedad. El mantenimiento de la salud no es un problema que deben solucionar los profesionales de la salud y la tecnología, la eficiencia y la eficacia de un sistema de salud dirigido al diagnostico y tratamiento de la enfermedad son los que permiten una mejora en la calidad de vida. 
La auditoria es de relevante importancia su incorporación es el sistema sanitario, por lo que es evidente que el usuario busca que lo atiendan con calidad y esto se lo logra con la atención de un buen servicio al cliente lo cual a la vez es fundamental dentro de la organización para alcanzar el éxito deseado, por lo que se debe propender a la innovación del servicio.

La auditoría médica es considerada como una herramienta de gestión clínica, no solo como medida de eficacia y eficiencia, sino también como sistema de información del comportamiento del usuario necesaria para la planeación. La responsabilidad médica consiste en la obligación del profesional de responder por eventuales daños producidos en su actuación. La responsabilidad puede ser de orden ético, moral, disciplinario, administrativo, civil y penal.

\section{Referencias Bibliográficas.}

Albrecht, K., \& Zemke, R., (1988), Gerencia del Servicio, Legis Editores, Bogotá, Colombia Alvarado Ramírez, Enrique E., (1986), Auditoría Médica. http://www.bvs.hn/RHP/pdf/1986/pdf/Vol10-1-1986-10.pdf

Álvarez, F., y Álvarez, A., (2009), Auditoría Médica y Epidemiología, Primera Edición, Ediciones ECOE, https://www.ecoeediciones.com/libros/sin\%20categorizar/ebook-auditoria-medica-yepidemiologia/

Atención centrada en el usuario, (s/f), Disponible en: calidad.salud.gob.mx/doctos/calidad/aten_usua.pdf

Berry, Leonard L., (2002), Un buen servicio ya no basta: cuatro principios de servicio excepcional al cliente, Editorial Norma, p. 365, Disponible en: http://goo.gl//VUyov

Cartwright, F. y Biddiss, M., (1972), Disease and history. Nueva York (NY): Thomas Crowell Company: 5-28.

Cifuentes, B., (2015), Auditoría del servicio, Revista CES Salud Pública, Universidad Cooperativa de Colombia, Volumen 6 N $^{\circ}$ 1: 100-113

Contraloría General de la República de Chile. (1996), Comité de Control Externo. Oficina Técnica: Normas de Auditoría de la Contraloría General, [Links]

Dussault, G., (1995), La epidemiología y la gestión de los servicios de salud, Boletín Epidemiológico OPS, 16(2): 1-5. 
Javier I. Haro-Alvarado; Hortencia Manuelita-Solorzano; Alba A. Sornoza-Pin; José M. Piguave-Reyes; María de los Ángeles Moreno-Cobos; Petita E. Pacheco-Castro

Garaycochea Cannon, V., (2000), Auditoría médica, Paediátrica, Vol. 3, No 1. http://sisbib.unmsm.edu.pe/bvrevistas/paediatrica/v03_n1/pdf/auditoria_medica.pdf

Ginocchio Vega, Carlos., (2011), Factores clave de la atención y servicio al cliente, Disponible en: https://www.slideshare.net/jhomar19/factores-clave-de-la-atencion-y-servicio-al-cliente

Guerrero, J. y Restrepo, P., et al., (2007), Pautas de Auditoría para el mejoramiento de la Calidad de la Atención en Salud, pp 1-71.

La Santa Biblia, (1960), Versión de Casiodoro de Reyna (1569), Sociedades Bíblicas Unidas, Buenos Aires: 39-71.

López, S., Garrido, F. y Hernández, M., (2000), Desarrollo histórico de la epidemiología: su formación como disciplina científica, salud pública de México /Vol.42, No.2.

Maizlish, N., (2000), Workplace health surveillance: an action-oriented approach, $1^{\text {a }}$ Ed. New York: Oxford University Press.

Manrique, J., (2015), Epidemiología y demografía en gestión en salud, Universidad Cayetano Heredia. https://www.slideshare.net/.../epidemiologa-y-demografa-en-gestin-en-salud48659167.

Ministerio de protección social, (2007), Pautas de Auditoría para el Mejoramiento de la Calidad de la Atención en Salud, Programa de Apoyo a la Reforma de Salud, editorial: Imprenta Nacional de Colombia, Bogotá, D. C.

Minsalud, (2013). Lineamiento para la auditoria de la calidad en la atención del paciente con diagnostico de dengue, Programa de promoción, prevención y control de las etv y zoonosis, Bogotá D.C., Colombia.

Moya, H.B., Venturini, R.G. y Baeza, G.A., (1968), Auditoría Médica. Servicio Nacional de Salud, Dirección General, Unidad de Auditoría Médica. [Links]

Orlando, A., (2005), Uso de la epidemiología en la planificación de los servicios de salud, Curso anual de auditoría médica Hospital Alemán, http://www.auditoriamedicahoy.com.ar/biblioteca/Epidemiologia_en_la_planificacion_de_lo s_Servicios_de_Salud.pdf

Osorio S., Guido, Sayes V., Nilda, Fernández M., Lautaro, Araya C., Ester y Poblete M., Dennis, (2002), Auditoria médica: herramienta de gestión moderna subvalorada, Rev. méd. Chile V.130 No 2, Santiago, http://dx.doi.org/10.4067/S0034-98872002000200014

Pulido, I., (2010), Breve Historia de la Epidemiología Ocupacional, http://ingridpulido.blogspot.com/2010/10/breve-historia-de-la-epidemiologia.html

Rantanen, J., Kauppinen, T., Toikkanen, J., Kurpa, K., Lehtinen, S., Leino, T., (2001), Work and health country profiles. Country profiles and national surveillance indicators in occupational health and safety. People and work research report 44. WHO / FIOH, 2001. Disponible en http://www.who.int/occupational_health/regions/en/oeheurcountryprofiles.pdf.

Rivadeneyra A., Artundo C., (2008), Hacia una nueva organización de los dispositivos de salud pública en España, Informe SESPAS 2008, Gac Sanit, 22 (Suppl 1): 96-103. 
Rodríguez, L., Lozano, S., (2012), Estudio de la satisfacción de los usuarios luego de la adopción del Sistema Único de Acreditación en Salud, en el Centro Policlínico del Olaya, Vol. 4, Núm. 1. ISSN: 2145-1389- e-ISSN: 2463-1140-DOI:https://doi.org/10.15332/s21451389.2012.0001.04

Solar, M.A., (1960), Auditoría Médica I.: Auditoría Médica. Rev Méd Chile 1960; 88: 499. [Links]

Urbaneja, F., Lijo, A., Cabrerizo, J., Idiazabal, J., Zubía, A. y Padilla, A., (2015), Vigilancia epidemiológica en el trabajo, Primera edición, OSALAN, Instituto Vasco de Seguridad y Salud laborales.

Winslow ECA. (1943), The conquest of epidemic disease, A chapter in the history of ideas, Madison, Princeton University Press, Wisconsin, 117-160. 